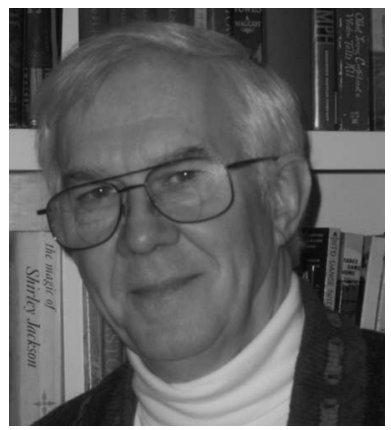

\section{Teaching Optical Engineering}

During the semester before I retired, I taught a course in optical design out of my textbook, Elements of Modern Optical Design. It turned out that I was not happy with the author's presentation, so I decided I had to rewrite, not revise, Elements. The biggest problem, as I saw it, was that the book was written in the early eighties and published in 1985 when ray tracing programs were just beginning to run on programmable calculators. At Georgia Tech we used a single Hewlett-Packard 9825 desktop calculator running OSLO.

Because it wasn't easy to adapt the ray tracing exercises on the desktop calculator for the textbook, I opted to use $y$ - $n u$ traces to introduce and explore the subject. As the spreadsheet became commonplace I used it as an alternative to the traditional "brick wall," a matrix format that was filled using the $y-n u$ trace calculations. Today, this "brick wall" and spreadsheet approach will not work with a student using a design program to learn to analyze an optical system. And the text needs to be completely rewritten with exercises and demonstrations that use current optical design programs. From my vantage point the exercises would be reminiscent of a series of columns, "Tracing Rays," that Mike Harrigan and I wrote for SPIE's OE Reports in 1991.

One problem with teaching ray tracing using computers is that there is always the "magical" element surrounding it. Introducing optical design programs to students is equivalent to asking the sorcerer's apprentice to clean up the lab. It is hard for beginning students to understand the results of their modifications to a system when they have nothing to compare them to. There is also the expenditure of time spent learning the application, which takes away from discussions of the optics and solving problems.

After the optical design course, I gave a special topics course that I described as "a lab course in optical design." My objective was to illustrate basic lens concepts, system analysis, and some optimization. One of my motivations for this course was to experiment with a new analysis tool just introduced by Optical Research Associates. It is an image simulation (IMS) feature that computes the image of a 2-D object including both aberrations and diffraction effects. This would let my students analyze a range of optical systems with the standard design analysis tools (ray fans, spot diagrams, modulation transfer functions, etc.) and see the results in relation to the visual performance of the systems. In addition to the obvious systems, air-spaced doublet, Cooke triplet, and the like, I also asked the students to examine the Hubble Space Telescope in its two incarnations and Newton's first telescope. For the last system, I teased its prescription out of several Royal Society papers that have been published on the device.

To reduce the time that students spend learning a program, I am constructing examples that incorporate macros while exploring basic lenses. Once students become comfortable with the concepts and operations of a design program, they can be directed to modify some of the lens specifications. Later, the students will be asked to enter or change pieces of the lens prescription until, finally, they can deal with an entire system.

In addition to lens analysis and design, Elements addressed Gaussian beams, scanning, modulation, spectrometry, and detection. Most design programs can simulate Gaussian beams, mechanical scanning, and spectrometry, but modulation and detection are more of a challenge. Apparently there are ways to simulate acousto-optic modulators in these programs, but I haven't played with them yet. As to which program should be used for this project, I'm not sure that only one will do. Right now I am trying to assess which features in the various design programs would best demonstrate the aspects of various optical systems. For example, there is no easy way in CODE V to reproduce OSLO's "slider wheel" feature, a useful tool for teaching optimization. After more than 20 years, I believe that a great deal of optical engineering can be demonstrated and taught on the computer. The challenge that I have set for myself is to prove it and publish the result. 